\title{
Primitive permutation groups satisfying the small orbit property and a problem of Bourgain and Kalai
}

\author{
Carmit Benbenisty ${ }^{1}$ (1) \\ Received: 1 November 2017 / Accepted: 30 September 2018 / Published online: 31 October 2018 \\ (c) The Author(s) 2018
}

\begin{abstract}
We classify the primitive but not affine permutation subgroups of $S_{n}$ with the following property: There exists some unbounded subset $X$ of size at most $\log n$ whose orbit is polynomially bounded (in terms of $n$ ). This class of subgroups arises naturally in the study of threshold behavior of $G$-symmetric properties of Boolean functions. Our result answers a problem of Bourgain and Kalai. Contrary to a suggestion in their paper, we give an example for a primitive permutation group with this property, which contains $A_{m}$ as a section for $m \rightarrow \infty$.
\end{abstract}

Keywords Permutation groups $\cdot$ Small orbit property $\cdot$ Primitive groups

\section{Introduction}

Let $I=\left\{n_{t} \mid t \in \mathbb{N}\right\}$ be a sequence of the natural numbers $\mathbb{N}$. For a sequence $G_{t} \leq S_{n_{t}}(t \in \mathbb{N})$ of primitive permutation groups, we say that $\left\{G_{t}\right\}$ has the small orbit property if there exist some constants $b$ and $c$ and some subsets $X_{t} \subseteq\left\{1, \ldots, n_{t}\right\}$ such that $\left\{\left|X_{t}\right|\right\}$ is not bounded, for all $t\left|X_{t}\right| \leq \log n_{t}$, and $\left|G_{t}\left(X_{t}\right)\right| \leq n_{t}^{b}$.

Consider the following example:

Let $G$ be $A_{n}$ or $S_{n}$ acting on $\Omega=\{1, \ldots, n\}(n \geq 3)$. Then, $G$ acts transitively on the set of all subsets of $\Omega$ of size $k$ for any $k$, and therefore, the size of the orbit of any subset $X$ of size $k$ is $\left(\begin{array}{l}n \\ k\end{array}\right)$. Clearly, the small orbit property is not satisfied.

A more interesting example is the case where $S_{n}$ acts on $\Omega=\left(\frac{\{1,2, \ldots, n\}}{2}\right)$ (namely all 2-subsets of $\{1, \ldots, n\}$ ), and in this case, the orbits correspond to isomorphism classes of graphs. Note that also here the small orbit property is not satisfied.

Let $G_{t}(t \in \mathbb{N})$ be a sequence of finite groups. Let $I=\left\{n_{t} \mid t \in \mathbb{N}\right\}$. Bourgain and Kalai posed the question of understanding primitive permutation groups with the

\footnotetext{
$\triangle$ Carmit Benbenisty

carmit.benbenishty@gmail.com

1 Kibbutzim College of Education Technology and the Arts, Tel Aviv, Israel
} 
small orbit property (see [1]). They proposed that the following are equivalent for an infinite set $I$ of natural numbers and a sequence of groups $G_{t}(t \in I)$ :

1. For any embedding of $G_{t}$ in $S_{n_{t}}$ such that the action on $\left\{1, \ldots, n_{t}\right\}$ is primitive, $G_{t}$ satisfies the small orbit property.

2. There exists some $M$, such that $G_{t}$ does not have $A_{m}$ as a composition factor for $m$ greater than $M$.

The motivation for studying permutation groups with the small orbit property comes from studies of threshold phenomena under symmetry and, in particular, a paper by Bourgain and Kalai [1].

Consider a monotone Boolean function $f\left(x_{1}, x_{2}, \ldots, x_{n}\right)$. Assume that $f$ is invariant under the action of some primitive group $G$. Let $\mu_{p}(f)$ be the probability that $f=1$ when every variable $x_{i}$ equals 1 with probability $p$ and equals 0 with probability $1-p$ (independently). Unless $f$ is a constant function, $\mu_{p}(f)$ is a strictly monotone function of $p$. Define the critical probability $p_{\mathrm{c}}=p_{\mathrm{c}}(f)$ by $\mu_{p_{\mathrm{c}}}(f)=1 / 2$. Let $\epsilon>0$ be a fixed tiny real number (say $\epsilon=1 / 1000$ ) and suppose that $\mu_{p_{1}}(f) \epsilon$ and $\mu_{p_{2}}(f)=1-\epsilon$. The interval $\left[p_{1}, p_{2}\right]$ is called the threshold interval for the Boolean function $f$. Some results of Bourgain, Friedgut and Kalai show that if $G$ satisfies the condition that the orbits of "large" subsets are "large," then the threshold is sharp (see [2]), i.e., $p_{2}-p_{1}=o(1)$ (as a function of $n$ ). Friedgut and Kalai [2] described an upper bound for the threshold interval for Boolean functions which is invariant under a transitive group $G$. Friedgut and Kalai gave an upper bound for the length of the threshold interval when the function is invariant under a transitive permutation group and showed that when $\log p_{\mathrm{c}} / \log n \rightarrow 0$, then the length of the threshold interval is actually $o\left(p_{\mathrm{c}}\right)$.

Bourgain and Kalai developed a method to show stronger results for permutation groups when there are no large sets with small orbits. They observed that no further improvement over the bounds given in [2] is possible when the group satisfies the small orbit property. This leads naturally to the question considered here.

In this paper, we give a classification of all primitive but not affine groups which satisfy the small orbit property. This classification shows that the two properties studied by Bourgain and Kalai are incomparable: namely, the first property of Bourgain and Kalai does not imply the second, and the second property does not imply the first.

Theorem Let $G_{t} \leq S_{n_{t}}$ be a sequence of primitive groups. Let $G=G_{t}$ for $t$ large enough. Assuming $G_{t}$ are not affine groups, the following two conditions hold: $\left\{G_{t}\right\}$ satisfies the small orbit property if and only iffor all sufficient large $t G_{t}$ is one of the following :

1. $G_{t} \leq S_{k_{t}} w r S_{m_{t}}$ (product type) where $m_{t}$ is not bounded.

2. $G_{t} \leq H_{t} w r S_{m_{t}}$ (product type) where $m_{t}$ is bounded and $H_{t}$ is a primitive permutation group satisfying the small orbit property.

3. $G_{t}$ is of diagonal type.

4. $G_{t}$ is of wreath product type.

5. $G_{t}$ is an almost simple exceptional group of Lie type.

6. $G_{t}$ is an almost simple classical group and any point stabilizer of $G_{t}$ is an irreducible group. 
7. $G_{t}$ is an almost simple classical group and $\operatorname{Soc}\left(G_{t}\right)=P \operatorname{SL}\left(k_{t}, q_{t}\right), \operatorname{PSp}\left(2 k_{t}, q_{t}\right)$, $P U\left(k_{t}, q_{t}^{2}\right), \quad P O\left(2 k_{t}+1, q_{t}\right), P O^{+}\left(2 k_{t}, q_{t}\right)$ or $P O^{-}\left(2 k_{t}, q_{t}\right)$, where $q_{t}$ is not bounded.

Remark We do not have a full characterization of affine groups that satisfying the small orbit property.

Remark In our proof, we show that if $\left\{G_{t}\right\}$ has the small orbit property, then there exist some subset $X_{t} \subseteq\left\{1, \ldots, n_{t}\right\}$ such that $\left\{\left|X_{t}\right|\right\}$ is not bounded, $\left|X_{t}\right| \leq \log n_{t}$ for all $t$ and $\left|G_{t}\left(X_{t}\right)\right| \leq n_{t}^{5}$.

In particular, the two properties of Bourgain and Kalai are incomparable. Indeed, the following are immediate consequences of the theorem.

Corollary 1 The sequence $S_{k_{t}} w r S_{m_{t}}$, where $m_{t}$ is not bounded, satisfies the first property of Bourgain and Kalai, but not the second.

Corollary 2 The sequence PSL $\left(k_{t}, q_{t}\right)$, where $q_{t}$ is bounded satisfies the second property of Bourgain and Kalai, but not the first.

\section{Proof of the theorem}

Recall that by the O'Nan Scott Theorem (see [4]) every finite primitive group $G$ is one of the following:

(1) $G$ is an almost simple group.

(2) $G \leq \operatorname{AGL}(V)$ where $V$ is a vector space of dimension $k$ over a field $F$ of size $q$.

(3) $G$ is of simple diagonal type.

(4) $G$ is of product type.

(5) $G$ is of twisted wreath product type.

Let $G_{t} \leq S_{n_{t}}$ be a sequence of primitive groups. Assuming $G_{t}$ is not of type (2), then $G_{t}$ must be of type (1), (3), (4) or (5). Whenever $G_{t}$ satisfies the small orbit property, we construct a subset $X_{t}$ whose size is not bounded but at $\operatorname{most} \log n_{t}$, but whose orbit size is less than $n_{t}{ }^{5}$. Otherwise, we give a proof that $G_{t}$ does not satisfy the small orbit property. Note that if $\left|G_{t}\right|<n_{t}{ }^{5}$, then we may take any subset $X_{t}$ such that $\left|X_{t}\right|$ is not bounded but of size at $\operatorname{most} \log n_{t}$ and obtain $\left|G_{t}\left(X_{t}\right)\right| \leq\left|G_{t}\right|<n_{t}^{5}$. Hence, in what follows, we may assume that $\left|G_{t}\right|>n_{t}{ }^{5}$.

Since by enlarging the group the size of the orbits cannot go down, then whenever $G_{t}$ has the small orbit property, we may replace $G_{t}$ by a maximal subgroup of $S_{n_{t}}$ of the same type.

Case 1 Assume that $G_{t}$ is an almost simple group. Then, by the classification theorem, either $\operatorname{Soc}\left(G_{t}\right)=A_{k_{t}}$ for some $k_{t}$, or $\operatorname{Soc}\left(G_{t}\right)$ is a simple group of Lie type, or $\operatorname{Soc}\left(G_{t}\right)$ is a sporadic simple group. By taking $t$ large enough, we may exclude the last case. So suppose now that $\operatorname{Soc}\left(G_{t}\right)$ is a Lie type group. We shall use the following. 
Lemma 1 Let $G \leq S_{n}$ be an almost simple exceptional group of Lie type. Suppose $G$ is primitive and $n$ is large enough. Then, $|G|<n^{5}$.

Proof Suppose first that $G$ is simple. Denote by $m(G)$ the minimal index of a proper subgroup of $G$. Estimates for $m(G)$ in the various cases are given in Lemma 6.4 of [6]. Comparing them to the known orders of the groups $G$, one easily sees that $|G|<m(G)^{5-\epsilon}$ for some fixed $\epsilon>0$. Since $m(G) \leq n$, this yields $|G|<n^{5-\epsilon}$ whenever $G$ is an exceptional simple transitive subgroup of $S_{n}$.

Now, if $G$ is not simple, let $K$ be its simple socle. Then, $K \leq S_{n}$ is transitive, so we have $|K| \leq n^{5-\epsilon}$. It is easy to see that $|\operatorname{Out}(K)| \leq \log |K|$ and $\operatorname{so}|\operatorname{Out}(K)| \leq$ $5 \log n<n^{\epsilon}$ for large enough $n$. It follows that $|G| \leq|K||\operatorname{Out}(K)|<n^{5-\epsilon} n^{\epsilon}=n^{\overline{5}}$, as required.

Thus, any exceptional almost simple group of Lie type satisfies the small orbit property. Assume now that $G_{t}$ is not exceptional. Hence, $G_{t}$ is an almost simple classical group. Moreover, the values of $\left|G_{t}\right|$ and $m\left(G_{t}\right)$ (see [3] pages 170,175) imply that $k_{t}$, the rank of $G_{t}$, is at least 3. Let $H_{t} \leq G_{t}$ be a point stabilizer. Assume first that $H_{t}$ is irreducible. By a theorem of M.Liebeck and J.Saxl [5], we have $\left|G_{t}\right| \leq n_{t}{ }^{5}$, and therefore, $G_{t}$ satisfies the small orbit property. Assume now that $H_{t}$ is a reducible group. Clearly, $H_{t}$ is a maximal subgroup of $G_{t}$, since $G_{t}$ is primitive. The maximal reducible subgroups of the classical groups are well known, and we shall use their description in constructing the required subset $X_{t}$.

Using the reducibility of the point stabilizer, it follows that there exist some $d_{t}, q_{t}$ and $k_{t} \geq 3$ such that $\operatorname{Soc}\left(G_{t}\right)$ is one of the following:

(A) a) $\operatorname{PSL}\left(k_{t}, q_{t}\right)$ acting on all $d_{t}$-dimensional subspaces of $V_{t}=\left(F_{q_{t}}\right)^{k_{t}}$.

b) $\operatorname{PSL}\left(k_{t}, q_{t}\right)$ acting on pairs of subspaces of complementary dimensions.

(B) a) $\operatorname{PSp}\left(2 k_{t}, q_{t}\right)$ acting on all $d_{t}$-dimensional non-degenerate subspaces of $V_{t}=\left(F_{q_{t}}\right)^{2 k_{t}}$.

b) $\operatorname{PSp}\left(2 k_{t}, q_{t}\right)$ acting on all $d_{t}$-dimensional totally singular subspaces of $V_{t}=\left(F_{q_{t}}\right)^{2 k_{t}}$.

c) $\operatorname{PSp}\left(2 k_{t}, q_{t}\right)$ acting on pairs of subspaces of complementary dimensions.

(C) a) $\mathrm{PU}\left(k_{t}, q_{t}{ }^{2}\right)$ acting on all $d_{t}$-dimensional non-degenerate subspaces of $V_{t}=\left(F_{q_{t}}{ }^{2}\right)^{k_{t}}$.

b) $\mathrm{PU}\left(k_{t}, q_{t}^{2}\right)$ acting on all $d_{t}$-dimensional totally singular subspaces of $V_{t}=\left(F_{q_{t}}{ }^{2}\right)^{k_{t}}$.

c) $\mathrm{PU}\left(k_{t}, q_{t}{ }^{2}\right)$ acting on pairs of subspaces of complementary dimension. 
(D) a)(1) $\mathrm{PO}\left(2 k_{t}+1, q_{t}\right)$ acting on all $d_{t}$-dimensional non-degenerate subspaces of $V_{t}=\left(F_{q_{t}}\right)^{2 k_{t}+1}$.

a)(2) $\mathrm{PO}\left(2 k_{t}+1, q_{t}\right)$ acting on all $d_{t}$-dimensional totally singular subspaces of $V_{t}=\left(F_{q_{t}}\right)^{2 k_{t}+1}$.

b)(1) $\mathrm{PO}^{+}\left(2 k_{t}, q_{t}\right)$ acting on all $d_{t}$-dimensional non-degenerate subspaces of $V_{t}=\left(F_{q_{t}}\right)^{2 k_{t}}$.

b)(2) $\mathrm{PO}^{+}\left(2 k_{t}, q_{t}\right)$ acting on all $d_{t}$-dimensional totally singular subspaces of $V_{t}=\left(F_{q_{t}}\right)^{2 k_{t}}$.

c)(1) $\mathrm{PO}^{-}\left(2 k_{t}, q_{t}\right)$ acting on all $d_{t}$-dimensional non-degenerate subspaces of $V_{t}=\left(F_{q_{t}}\right)^{2 k_{t}}$.

c)(2) $\mathrm{PO}^{-}\left(2 k_{t}, q_{t}\right)$ acting on all $d_{t}$-dimensional totally singular subspaces of $V_{t}=\left(F_{q_{t}}\right)^{2 k_{t}}$.

c)(3) $\mathrm{PO}\left(2 k_{t}+1, q_{t}\right)$ where $q_{t}$ is even and $G_{t}$ acts on all one-dimensional non-singular subspaces.

d) $\mathrm{PO}\left(2 k_{t}, q_{t}\right)$ or $\mathrm{PO}\left(2 k_{t}+1, q_{t}\right)$ acting on pairs of subspaces of complementary dimensions.

Note that any sequence $G_{t}$ of almost simple classical groups with the above action has the small orbit property if and only if its socle has this property. This follows from the inequality:

$$
\left|G_{t}\left(X_{t}\right)\right| \leq\left|\operatorname{Soc}\left(G_{t}\right)\left(X_{t}\right)\right|\left|\operatorname{Out}\left(G_{t}\right)\right| \leq\left|\operatorname{Soc}\left(G_{t}\right)\right| \cdot n_{t} .
$$

Therefore, we may restrict to the socle and assume $G_{t}$ is simple. So let $G_{t}$ be a sequence of simple classical groups with one of the actions above. We will show that if $q_{t}$ is not bounded, then $G_{t}$ satisfies the small orbit property, and if $q_{t}$ is bounded, then $G_{t}$ does not satisfy the small orbit property. Assume first that $q_{t}$ is not bounded. Suppose first that $G_{t}=P G L_{k_{t}}\left(q_{t}\right)$. Let $\left\{v_{t, 1}, \ldots, v_{t, k_{t}}\right\}$ be a basis for $V_{t}$ and let $H_{t} \leq G_{t}$ be a point stabilizer. Then, $H_{t}$ is a maximal subgroup of $G_{t}$. Assume first that $G_{t}$ acts on the set of all $d_{t}$-dimensional subspaces of $F_{q_{t}}{ }^{k_{t}}$ for

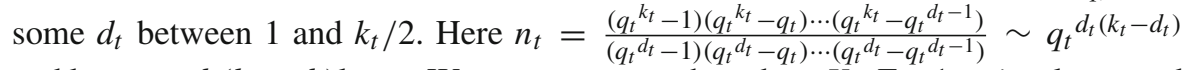
and $\log n_{t} \sim d_{t}\left(k_{t}-d_{t}\right) \log q_{t}$. We now construct the subset $X_{t}$. For $1 \leq i \leq \log q_{t}$ and $a_{t, 1}, \ldots, a_{t, \log q_{t}} \in F_{q_{t}}$, let

$$
U_{t, i}=\operatorname{Sp}\left(v_{t, 1}, \ldots, v_{t, d_{t}-1}, v_{t, d_{t}}+a_{t, i} v_{t, d_{t}+1}\right)
$$

Let

$$
X_{t}=\left\{U_{t, 1}, \ldots, U_{t, \log q_{t}}\right\}
$$

Then, $\log q_{t}-1 \leq\left|X_{t}\right| \leq \log q_{t} \leq \log n_{t}$, and since $q_{t}$ is not bounded, $\left|X_{t}\right|$ is not bounded as well. It is easy to see that any $A_{t} \in \mathrm{GL}_{k_{t}}\left(q_{t}\right)$ which stabilizes the subspace $\operatorname{Sp}\left(v_{t, 1}, \ldots, v_{t, d_{t}-1}\right)$ and the vectors $v_{t, d_{t}}, v_{t, d_{t}+1}$ also stabilizes $X_{t}$, and therefore, 


$$
\begin{aligned}
\left|G_{t X_{t}}\right| \geq & \left.\left(q_{t}^{d_{t}-1}-1\right)\left(q_{t}^{d_{t}-1}-q_{t}\right) \cdots q_{t}{ }^{d_{t}-1}-q_{t}{ }^{d_{t}-2}\right) \\
& \left(q_{t}^{k_{t}-\left(d_{t}+1\right)}-1\right) \cdots\left(q_{t}^{k_{t}-\left(d_{t}+1\right)}-q_{t}^{k_{t}-d_{t}}\right)
\end{aligned}
$$

It follows that

$$
\left|G_{t}\left(X_{t}\right)\right|=\frac{\left(q_{t}{ }^{k_{t}}-1\right)\left(q_{t}{ }^{k_{t}}-q_{t}\right) \cdots\left(q_{t}{ }^{k_{t}}-q_{t}{ }^{k_{t}-1}\right)}{\left|G_{t X_{t}}\right|} \leq n_{t}{ }^{2} .
$$

Thus, $G_{t}$ satisfies the small orbit property.

Assume now that $G_{t}$ acts on the set of all pairs of subspaces $\left(U_{t}, W_{t}\right)$ such that $U_{t} \oplus W_{t}=V_{t}$ and $\operatorname{dim} U_{t}=d_{t}$, where $d_{t} \leq k_{t} / 2$. Then, $n_{t}=q_{t}{ }^{2 d_{t}\left(k_{t}-d_{t}\right)}$ and $\log n_{t}=2 d_{t}\left(k_{t}-d_{t}\right) \log q_{t}$. We now construct the subset $X_{t}$. For $1 \leq i \leq \log q_{t}$ and $a_{t, 1}, \ldots, a_{t, \log q_{t}} \in F_{q_{t}}$, let

$$
U_{t, i}=\operatorname{Sp}\left(v_{t, 1}, \ldots, v_{t, d-1}, v_{t, d_{t}}+a_{t, i} v_{t, d_{t}+1}\right)
$$

and let

$$
W_{t}=\operatorname{Sp}\left(v_{t, d_{t}}, v_{t, d_{t}+2}, \ldots, v_{t, k_{t}}\right)
$$

Let

$$
X_{t}=\left\{U_{t, 1} \oplus W_{t}, \ldots, U_{t, \log q_{t}} \oplus W_{t}\right\}
$$

It is easy to see that any $A_{t} \in \mathrm{GL}_{k_{t}}\left(q_{t}\right)$ which stabilizes the $\operatorname{subspace} \operatorname{Sp}\left(v_{t, 1}, \ldots\right.$, $\left.v_{t, d_{t}-1}\right)$, the vectors $v_{t, d_{t}}, v_{t, d_{t}+1}$ and the subspace $\operatorname{Sp}\left(v_{t, d_{t}+2}, \ldots, v_{t, k_{t}}\right)$ also stabilizes $X_{t}$, and therefore,

$$
\begin{aligned}
\left|\left(G_{t}\right)_{X_{t}}\right| \geq & \left(q_{t}{ }^{d_{t}-1}-1\right)\left(q_{t}{ }^{d_{t}-1}-q_{t}\right) \cdots\left(q_{t}{ }^{d_{t}-1}-q_{t}{ }^{d_{t}-2}\right) \\
& \left(q_{t}{ }^{k_{t}-\left(d_{t}+1\right)}-1\right) \cdots\left(q_{t}{ }^{k_{t}-\left(d_{t}+1\right)}-q_{t}{ }^{k_{t}-d_{t}}\right)
\end{aligned}
$$

It follows that

$$
\left|G_{t}\left(X_{t}\right)\right|=\frac{\left(q_{t}^{k_{t}}-1\right)\left(q_{t}^{k_{t}}-q_{t}\right) \cdots\left(q_{t}^{k_{t}}-q_{t}^{k_{t}-1}\right)}{\left|G_{t X_{t}}\right|} \leq n_{t} .
$$

Thus, $G_{t}$ satisfies the small orbit property.

Assume now that $G_{t}$ acts on the set of all pairs of subspaces $\left(U_{t}, W_{t}\right)$ such that $U_{t} \oplus W_{t}, \operatorname{dim} U_{t}=d_{t}$, where $d_{t} \leq k_{t} / 2$. Then, $n_{t}=q_{t} d_{t}\left(2 k_{t}-3 d_{t}\right)$ and $\log n_{t}=d_{t}\left(2 k_{t}-\right.$ $\left.3 d_{t}\right) \log q_{t}$. We now construct the subset $X_{t}$. For $1 \leq i \leq \log q_{t}$ and $a_{t, 1}, \ldots, a_{t, \log q_{t}}$, let

$$
U_{t, i}=\operatorname{Sp}\left(v_{t, 1}, \ldots, v_{t, d_{t}-1}, v_{t, d_{t}}+a_{t, i} v_{t, d+1}\right)
$$


and let

$$
W_{t}=\operatorname{Sp}\left(v_{t, d_{t}}, v_{t, d_{t}+2}, \ldots v_{t, k_{t}}\right) \text {. }
$$

Let

$$
X_{t}=\left\{\left(U_{t, 1}, W_{t}\right), \ldots,\left(U_{t, \log q_{t}}, W_{t}\right)\right\}
$$

It is easy to see that any $A_{t} \in \mathrm{GL}_{k_{t}}\left(q_{t}\right)$ which stabilizes the subspace $\operatorname{Sp}\left(v_{t, 1}, \ldots\right.$, $\left.v_{t, d_{t-1}}\right)$, the vectors $v_{t, d}, v_{t, d_{t+1}}$ and the subspace $\operatorname{Sp}\left(v_{t, d_{t+2}}, \ldots, v_{t, k_{t}}\right)$ also stabilizes $X_{t}$, and therefore,

$$
\begin{aligned}
\left|\left(G_{t}\right)_{X_{t}}\right| \geq & \left(q_{t}{ }^{d_{t}-1}-1\right)\left(q_{t}{ }^{d_{t}-1}-q_{t}\right) \cdots\left(q_{t}{ }^{d_{t}-1}-q_{t}{ }^{d_{t}-2}\right) \\
& \left(q_{t}{ }^{k_{t}-\left(d_{t}+1\right)}-1\right) \cdots\left(q_{t}{ }^{k_{t}-\left(d_{t}+1\right)}-q_{t}{ }^{k_{t}-d_{t}}\right) .
\end{aligned}
$$

It follows that

$$
\left|G_{t}\left(X_{t}\right)\right|=\frac{\left(q_{t}^{k_{t}}-1\right)\left(q_{t}^{k_{t}}-q_{t}\right) \cdots\left(q_{t}{ }^{k_{t}}-q_{t}{ }^{k_{t}-1}\right)}{\left|\left(G_{t}\right)_{X_{t}}\right|} \leq n_{t}
$$

Thus, $G_{t}$ satisfies the small orbit property.

Assume now that $G_{t}$ acts on the set of all pairs of subspaces $\left(U_{t}, W_{t}\right)$ such that $U_{t} \subseteq$ $W_{t}, \operatorname{dim} U_{t}=d_{t}$, and $\operatorname{dim} W_{t}=k_{t}-d_{t}$, where $d_{t} \leq k_{t} / 2$. Then, $n_{t}=q_{t} d_{t}\left(2 k_{t}-3 d_{t}\right)$ and $\log n_{t}=d_{t}\left(2 k_{t}-3 d_{t}\right) \log q_{t}$. We now construct the subset $X_{t}$. Let $\left\{v_{1}, \ldots, v_{k}\right\}$ be a basis for $V$. We now construct the subset $X$. For $1 \leq i \leq \log q_{t}$ and $a_{t, 1}, \ldots, a_{t, \log q_{t}}$, let

$$
U_{t, i}=\operatorname{Sp}\left(v_{t, 1}, \ldots, v_{t, d_{t-1}}, v_{t, d_{t}}+a_{t, i} v_{t, d_{t+1}}\right)
$$

and let

$$
W_{t}=\operatorname{Sp}\left(v_{t, 1}, \ldots, v_{t, d_{t}-1}, v_{t, d_{t}}, v_{t, d_{t}+1}, v_{t, d_{t}+2}, \ldots, v_{t, k_{t}-d_{t}}\right)
$$

Let

$$
X_{t}=\left\{\left(U_{t, 1}, W_{t}\right), \ldots,\left(U_{t, \log q_{t}}, W_{t}\right)\right\}
$$

It is easy to see that for any $A_{t} \in \operatorname{GL}_{k_{t}}\left(q_{t}\right)$ which stabilizes the subspace $\operatorname{Sp}\left(v_{t, 1}, \ldots, v_{t, d_{t}-1}\right)$, the vectors $v_{t, d}, v_{d_{t}+1}$ and the subspace $\operatorname{Sp}\left(v_{t, d_{t}+2}, \ldots, v_{t, k_{t}}\right)$ also stabilizes $X_{t}$, and therefore,

$$
\begin{aligned}
\left|\left(G_{t}\right)_{X_{t}}\right| \geq & \left(q_{t}{ }^{d_{t}-1}-1\right)\left(q_{t}{ }^{d_{t}-1}-q_{t}\right) \cdots\left(q_{t}{ }^{d_{t}-1}-q_{t}{ }^{d_{t}-2}\right) \\
& \left(q_{t}{ }^{k_{t}-\left(d_{t}+1\right)}-1\right) \cdots\left(q_{t}{ }^{k_{t}-\left(d_{t}+1\right)}-q_{t}{ }^{k_{t}-d_{t}}\right) .
\end{aligned}
$$


It follows that

$$
\left|G_{t}\left(X_{t}\right)\right|=\frac{\left(q_{t}^{k_{t}}-1\right)\left(q_{t}^{k_{t}}-q_{t}\right) \cdots\left(q_{t}^{k_{t}}-q_{t}^{k_{t}-1}\right)}{\left|\left(G_{t}\right)_{X_{t}}\right|} \leq n_{t}{ }^{2} .
$$

Thus, $G_{t}$ satisfies the small orbit property.

Assume now that $G_{t}$ is any group not of types $D(c)(3)$ or $A$. Assume first that $G_{t}$ acts on the set of all $d_{t}$-dimensional totally singular subspaces for some $d_{t}$ between 1 and $k_{t} / 2-1$. Let $\left\{e_{t, 1}, f_{t, 1}, \ldots, e_{t, k_{t} / 2}, f_{t, k_{t} / 2}\right\}$ be a standard basis for $V_{t}$, i.e., $\left(e_{t, i}, e_{t, j}=\left(f_{t, i}, f_{t, j}=1\right.\right.$, and $\left(e_{t, i}=f_{t, j}=\delta_{i, j}\right.$ (it might be the case that instead of $e_{t, k_{t} / 2}, f_{t, k_{t} / 2}$ we will have basis elements $x_{t}, y_{t}$, with different relations, or that the dimension is odd, and we will have an additional basis element $x_{t}$, (see chapter 2 of [3]), but for our purpose it will not make a difference). For $1 \leq i \leq \log q_{t}$ and $a_{t, 1}, \ldots, a_{t, \log q_{t}}$, let

$$
U_{t, i}=\operatorname{Sp}\left(e_{t, 1}, \ldots, e_{t, d_{t}-1}, e_{t, d_{t}}+a_{t, i} e_{t, d_{t}+1}\right)
$$

Let

$$
X_{t}=\left\{U_{t, 1}, \ldots, U_{t, \log q_{t}}\right\}
$$

It is easy to see that any $A_{t} \in G_{t}$ which stabilizes the $\operatorname{subspace} \operatorname{Sp}\left(e_{t, d_{t}}, e_{t, d_{t}+1}\right)$ also stabilizes $X_{t}$. Using the results of chapter 2 of [3], we get that $\left|G_{t}\left(X_{t}\right)\right| \leq n_{t}{ }^{2}$. Assume now that $d_{t}=k_{t} / 2$. For $1 \leq i \leq \log q_{t}$ and $a_{t, 1}, \ldots, a_{t, \log q_{t}} \in F_{q_{t}}$, let

$$
U_{t, i}=\operatorname{Sp}\left(e_{t, 1}, \ldots, e_{t, k_{t} / 2-2}, e_{k / 2-1}+a_{i} f_{k / 2}, e_{k / 2}-a_{i} f_{k / 2-1}\right)
$$

(in the symplectic case, we take $e_{t, k_{t} / 2}+a_{t, i} f_{t, k_{t} / 2-1}$ instead of $e_{t, k_{t} / 2}-a_{t, i} f_{t, k_{t} / 2-1}$ ). Let

$$
X_{t}=\left\{U_{t, 1}, \ldots, U_{t, \log q_{t}}\right\}
$$

As before, we get that $\left|G_{t}\left(X_{t}\right)\right| \leq n_{t}{ }^{2}$. Assume now that $G_{t}$ acts on the set of all $d_{t}$-dimensional non-degenerate subspaces of $V_{t}$. For $1 \leq i \leq \log q_{t}$ and $a_{t, 1}, \ldots, a_{t, \log q_{t}} \in F_{q_{t}}$, let

$$
U_{t, i}=\left\{e_{t, 1}, f_{t, 1}, \ldots, e_{t, d_{t} / 2-1}, f_{t, d_{t} / 2-1}, e_{t, d_{t} / 2}, f_{t, d_{t} / 2}+a_{t, i} e_{t, d_{t} / 2+1}\right\} .
$$

Let

$$
X_{t}=\left\{U_{t, 1}, \ldots, U_{t, \log q_{t}}\right\}
$$

Thus, we get that $\left|G_{t}\left(X_{t}\right)\right| \leq n_{t}{ }^{2}$. Assume now that $G_{t}$ is of type $D(c)$ (3) above. Let $\left\{e_{t, 1}, f_{t, 1}, \ldots, e_{t, k_{t} / 2}, f_{t, k_{t} / 2}\right\}$ be a symplectic basis for $V_{t}$. For $1 \leq i \leq \log q_{t}$ and $a_{t, 1}, \ldots, a_{t, \log q_{t}}$, let

$$
U_{t, i}=\operatorname{Sp}\left(e_{t, 1}+a_{t, i} f_{t, 1}\right\}
$$


Let

$$
X_{t}=\left\{U_{t, 1}, \ldots, U_{t, \log q_{t}}\right\}
$$

It follows that $\left|G_{t}\left(X_{t}\right)\right| \leq n_{t}{ }^{2}$. Hence, whenever $q_{t}$ is not bounded, $G_{t}$ satisfies the small orbit property. Assume now that $q_{t}$ is bounded. Let $G_{t}$ be any group of types $A-D$ above. We will show that under the action of $G_{t}$ on the set of all onedimensional totally singular subspaces of $V_{t}$, the size of the orbit of any unbounded subset of size at most $c \log n_{t}$ is not polynomial. Let $X_{t}$ be any unbounded set of vectors of size at most $c \log n_{t}$. Let $r_{t}=\operatorname{dim} \operatorname{Span}\left(X_{t}\right)$. Clearly, $r_{t}$ is not bounded (since $q_{t}$ is bounded). Let $\left\{x_{t, 1}, \ldots, x_{t, r_{t}}\right\} \subseteq X_{t}$ be a base for $\operatorname{Span}\left(X_{t}\right)$, and $\left\{v_{t, r_{t}+1}, \ldots, v_{t, k_{t}}\right\}$ its complement to a base for $V_{t}$. Let $g_{t} \in\left(G_{t}\right)_{X_{t}}$. Then, for all $1 \leq i \leq r_{t} g_{t}\left(x_{t, i}\right) \in X_{t}$ and $g_{t}\left(v_{t, r_{t+i}}\right) \notin \operatorname{Span}\left(X_{t}\right)$. Therefore,

$$
\begin{aligned}
\left|G_{t X_{t}}\right| & \leq\left|X_{t}\right|\left(\left|X_{t}\right|-1\right) \cdots\left(\left|X_{t}\right|-\left(r_{t}-1\right)\right)\left(q_{t}{ }^{k_{t}}-q^{t^{r_{t}}}\right) \cdots\left(q_{t}{ }^{k_{t}}-q_{t}{ }^{k_{t}-1}\right) \\
& \leq\left|X_{t}\right|^{r_{t}}\left(q_{t}{ }^{k_{t}}-q_{t}{ }^{r_{t}}\right) \cdots\left(q_{t}{ }^{k_{t}}-q_{t}{ }^{k_{t}-1}\right) .
\end{aligned}
$$

It follows that

$$
\begin{aligned}
& \left|G_{t}\left(X_{t}\right)\right|=\left|G_{t}:\left(G_{t}\right)_{X_{t}}\right| \geq \frac{\left(q_{t}{ }^{k_{t}}-1\right) \cdots\left(q_{t}{ }^{k_{t}}-q_{t}{ }^{k_{t}-1}\right)}{\left|X_{t}\right|^{r_{t}}\left(q_{t}{ }^{k_{t}}-q_{t}{ }^{r_{t}}\right) \cdots\left(q_{t}{ }^{k_{t}}-q_{t}{ }^{k_{t}-1}\right)} \\
& =\frac{\left(q_{t}^{k_{t}}-1\right) \cdots\left(q_{t}^{k_{t}}-q_{t}^{r_{t}-1}\right)}{\left|X_{t}\right|^{r_{t}}} \geq \frac{\left(q_{t}^{k_{t}}-q_{t}{ }^{r_{t}-1}\right)^{r_{t}}}{\left(c k_{t} \log q_{t}\right)^{r_{t}}} \geq \frac{\left(q_{t}{ }^{k_{t}-1}\right)^{r_{t}}}{\left(c k_{t} \log q_{t}\right)^{r_{t}}} \\
& =\left(\frac{\left(q_{t}^{k_{t}-1}\right)}{c k_{t} \log q_{t}}\right)^{r_{t}}>\left(\frac{\left(q_{t}^{k_{t}-2}\right)}{c k_{t}}\right)^{r_{t}} \text {. }
\end{aligned}
$$

Since $r_{t}$ is not bounded, it now follows that $\left|G_{t}\left(X_{t}\right)\right|$ is not polynomial in $n_{t}=q_{t}{ }^{k_{t}}$, as desired.

Case 3 Assume that $G_{t}$ is of diagonal type. We will show that $G_{t}$ satisfies the small orbit property. We may assume that there exists some integer $k_{t}$ such that $G_{t}=H_{t}{ }^{k_{t}}$. (Out $\left.\left(H_{t}\right) \times S_{k_{t}}\right)$, where $H_{t}$ is a non-abelian simple group, $k_{t}$ is a positive integer and $G_{t}$ acts on the set of all cosets of the diagonal group $D_{t}=\left\{\left(h_{t}, \ldots, h_{t}\right)\right.$ : $\left.h_{t} \in H_{t}\right\}$ Denote this set by $\Omega$. Here $n_{t}=\left|H_{t}{ }^{k_{t}-1}\right|$ and $\log n_{t}=\left(k_{t}-1\right) \log \left|H_{t}\right|$. Since $\left|G_{t}\right|=\left|H_{t}{ }^{k_{t}}\right|\left|H_{t}\right| k_{t} ! \leq n_{t}{ }^{2} k_{t}$ !, then we may assume that $k_{t}$ is not bounded. Let $L_{t}<H_{t}$ be a nontrivial subgroup of $H_{t}$ and let $1 \neq l_{t} \in L_{t}$. Let

$$
X_{t}=\left\{\left(l_{t}, 1, \ldots, 1\right) D_{t},\left(1, l_{t}, 1, \ldots, 1\right) D_{t}, \ldots,\left(1, \ldots, 1, l_{t}\right) D_{t}\right\}
$$

Then, $\left|X_{t}\right|=k_{t} \leq \log n_{t}$. Since $\left|S_{k_{t}}\left(X_{t}\right)\right|=1$ we have

$$
\left|G_{t}\left(X_{t}\right)\right|=\left|H_{t}{ }^{k_{t}} \cdot\left(\operatorname{Out}\left(H_{t}\right) \times S_{k_{t}}\right)\left(X_{t}\right)\right| \leq n_{t}{ }^{2}\left|S_{k_{t}}\left(X_{t}\right)\right|=n_{t}{ }^{2} .
$$

Case 4 Assume that $G_{t}$ is of product type. Let $H_{t}$ be a primitive permutation group of type (1) or (3) acting on a set $\Gamma_{t}=\left\{1, \ldots, k_{t}\right\}$. For $m_{t}>1$, let $W_{t}=H_{t} w r S_{m_{t}}$ 
and take $W_{t}$ to act on $\Omega_{t}=\Gamma_{t}{ }^{m_{t}}$ in its natural product action. Then, $G_{t} \leq H_{t} w r S_{m_{t}}$, and $n_{t}=k_{t}{ }^{m_{t}}$. Assume first that $m_{t}$ is not bounded. We will show that $G_{t}$ satisfies the small orbit property. Let $x_{t}, y_{t} \in \Gamma_{t}$ and let

$$
X_{t}=\left\{\left(x_{t}, y_{t}, \ldots, y_{t}\right),\left(y_{t}, x_{t}, y_{t}, \ldots, y_{t}\right), \ldots,\left(y_{t}, \ldots, y_{t}, x_{t}\right)\right\}
$$

Then, $\left|X_{t}\right|=m_{t} \leq \log n_{t}$. It is easy to see that $\left|S_{k_{t}}{ }^{m_{t}}\left(X_{t}\right)\right| \leq\left(k_{t}{ }^{m_{t}}\right)^{2}=n_{t}{ }^{2}$. Since $\left|S_{m_{t}}\left(X_{t}\right)\right|=1$, we have $\left|G_{t}\left(X_{t}\right)\right| \leq\left|S_{k_{t}} m_{t} \cdot S_{m_{t}}\left(X_{t}\right)\right| \leq n_{t}{ }^{2}\left|S_{m_{t}}\left(X_{t}\right)\right|=n_{t}{ }^{2}$. Assume now that $m_{t}$ is bounded. We will show that $G_{t}$ has the small orbit property if and only if $H_{t}$ has the small orbit property: Assume first that $H_{t}$ satisfies the small orbit property. As we have shown (see cases 1 and 3), there exists some subset $Y_{t}$ which is not bounded but of size at most $\log k_{t}$ such that $\left|H_{t}\left(Y_{t}\right)\right| \leq k_{t}{ }^{5}$. Denote $Y_{t}=\left\{y^{t}{ }_{1}, \ldots, y^{t}{ }_{\left|Y_{t}\right|}\right\}$. Let

$$
X_{t}=\left\{\left(y_{1}^{t}, \ldots, y_{1}^{t}\right),\left(y_{2}^{t}, \ldots, y_{2}^{t}\right), \ldots,\left(y^{t}{ }_{\left|Y_{t}\right|}, \ldots y_{\left|Y_{t}\right|}^{t}\right)\right\}
$$

Then, $X_{t}$ is not bounded but of size at most $\log n_{t}$. Since $\left|H_{t}\left(Y_{t}\right)\right| \leq k_{t}{ }^{5}$, then $\left|H_{t}{ }^{m_{t}}\left(X_{t}\right)\right| \leq\left(k_{t}^{5}\right)^{m_{t}}=\left(k_{t}^{m_{t}}\right)^{5}=n_{t}{ }^{5}$. Clearly, $\left|S_{m_{t}}\left(X_{t}\right)\right|=1$, and therefore, $\left|H_{t}^{m_{t}} \cdot S_{m_{t}}\left(X_{t}\right)\right| \leq n_{t}^{5}$.

Assume now that $H_{t}$ does not satisfy the small orbit property. By our classification (see cases 1 and 3 ), $H_{t}$ must be an almost simple group. A previous argument (see case 1) implies that $\operatorname{Soc}\left(H_{t}\right)$ does not satisfy the small orbit property as well. We will show now that $G_{t}$ does not satisfy the small orbit property as well: Let $X_{t}$ be any unbounded subset of $\Omega_{t}$ of size at most $c \log n_{t}$. Denote by $X^{t}{ }_{i}$ the projection on the $i^{\prime} t h$ axis. Since $m_{t}$ is bounded, then there exists some $1 \leq i \leq m_{t}$ such that $X^{t}{ }_{i}$ is an unbounded set of size at most $c m_{t} \log k_{t}$. Hence, under our assumption $\left|\left(\operatorname{Soc}\left(H_{t}\right)\right)\left(X^{t}{ }_{i}\right)\right|$ is not polynomial in $k_{t}$, and since $m_{t}$ is bounded, then $\left|\left(\operatorname{Soc}\left(H_{t}\right)\right)\left(X^{t}{ }_{i}\right)\right|$ is not polynomial in $n_{t}$. It now follows that $\left|\left(\operatorname{Soc}\left(H_{t}\right)\right)^{m_{t}}\left(X_{t}\right)\right|$ is not polynomial in $n_{t}$. Since $\left(\operatorname{Soc}\left(H_{t}\right)\right)^{m_{t}} \leq G_{t}$, then we get now that $\left|G_{t}\left(X_{t}\right)\right|$ is not polynomial in $n_{t}$, as desired.

Case 5 Any group $G_{t}$ of type 5 satisfies the small orbit property, since any twisted wreath product group is contained in a group of product type of the form $H_{t} w r S_{m_{t}}$ where $H_{t}$ is of diagonal type (see [4]) .

Acknowledgements The author is grateful to Aner Shalev and Gil Kalai for their generous help and encouragement.

Open Access This article is distributed under the terms of the Creative Commons Attribution 4.0 International License (http://creativecommons.org/licenses/by/4.0/), which permits unrestricted use, distribution, and reproduction in any medium, provided you give appropriate credit to the original author(s) and the source, provide a link to the Creative Commons license, and indicate if changes were made.

\section{References}

1. Bourgain, J., Kalai, G.: Influences of variables and threshold intervals under group symmetries. Geom. Funct. Anal. 7, 438-461 (1997) 
2. Friedgut, E., Kalai, G.: Every monotone graph property has a sharp threshold. Proc. Amer. Math. Soc. 124, 2993-3002 (1996)

3. Kleidman, Peter, Liebeck, Martin: The Subgroup Structure of the Finite Classical Groups, vol. 129. Cambridge University Press, Cambridge (1990)

4. Liebeck, M.W., Praeger, C.E., Saxl, J.: On the O'Nan-Scott theorem for finite primitive groups. J. Aust. Math. Soc. 44, 389-396 (1988)

5. Liebeck, M.W., Saxl, J.: Maximal subgroups of finite simple groups and their automorphism groups. Contemp. Math. 131(1), 243-259 (1992)

6. Liebeck, M.W., Shalev, A.: Simple groups, probabilistic methods, and a conjecture of Kantor and Lubotzky. J. Algebra 184, 31-57 (1996) 